\title{
Data-efficient Bayesian verification of parametric Markov chains
}

\author{
E. Polgreen ${ }^{1}$, V.B. Wijesuriya ${ }^{1}$, S. Haesaert ${ }^{2}$, and A. Abate ${ }^{1}$ \\ 1 Department of Computer Science, University of Oxford \\ 2 Department of Electrical Engineering, Eindhoven University of Technology
}

\begin{abstract}
Obtaining complete and accurate models for the formal verification of systems is often hard or impossible. We present a data-based verification approach, for properties expressed in a probabilistic logic, that addresses incomplete model knowledge. We obtain experimental data from a system that can be modelled as a parametric Markov chain. We propose a novel verification algorithm to quantify the confidence the underlying system satisfies a given property of interest by using this data. Given a parameterised model of the system, the procedure first generates a feasible set of parameters corresponding to model instances satisfying a given probabilistic property. Simultaneously, we use Bayesian inference to obtain a probability distribution over the model parameter set from data sampled from the underlying system. The results of both steps are combined to compute a confidence the underlying system satisfies the property. The amount data required is minimised by exploiting partial knowledge of the system. Our approach offers a framework to integrate Bayesian inference and formal verification, and in our experiments our new approach requires one order of magnitude less data than standard statistical model checking to achieve the same confidence.
\end{abstract}

\section{Introduction}

Complex engineering systems, such as autonomous vehicles, are often safetycritical and demand high guarantees of correctness. Given a complete model of the system of interest, these guarantees can be obtained through formal methods, such as model checking [1], though the outcomes of these formal proofs are bound to the model of the system of interest. Obtaining a complete model is not possible for systems with uncertain stochastic dynamics, but we can capture these dynamics with parameterised Markov chains. Model checking now produces a result dependent on knowledge of the value of parameters within the model.

In this work we integrate the use of model checking techniques (for parameter synthesis over the model) with data-based approaches (for parametric Bayesian inference), in order to compute a confidence, based on observed data collected from the system, that the system satisfies a given specification.

The proposed approach is distinctively different from statistical model checking (SMC) [14], a known data-based technique for model verification, and has a distinct set-up and addresses a different objective: The original SMC tools such 
as YMer and Vesper target systems with fully known models too large for conventional model checking, and use the known models to generate simulated data; SMC has also been applied in a model-free setting where system-generated data is directly employed towards statistical validation of properties of interest [19]. Our technique instead targets partially known systems, captured as a parameterised model class, and still uses data collected from the original system.

In general SMC requires a large amount of sample data covering the entire system behaviour to obtain good confidence results, our method requires much less sample data, and can accommodate data with only partial coverage.

Our method is elucidated in three phases. In the first phase, having a parameterised model of our partially known system, we use parameter synthesis to determine a set of feasible parameters over the given model class, namely those parameters corresponding to models of the system satisfying the given specification. Among a number of alternatives, we use an existing parameter synthesis method implemented in PRISM [11]. The second phase which, executed in parallel with the first, uses Bayesian statistics to infer a distribution over the likely values of the parameters of the model class, based on data collected from the underlying system. Finally, we combine the outputs from the previous two phases to compute the confidence attached to the system satisfying the given specification.

Alongside the new methodology introduced in this work (first presented over different model class and properties in [9]), the key contribution resides in phase two: our algorithm introduces expansions of states and transitions of the parameterised Markov chain, which guarantees the posterior probability distributions over the parameters can be obtained analytically, and integrated easily. The work discusses a case study, demonstrating the implementation of the algorithm, and a comparison with a standard SMC procedure.

Related Work Statistical Model Checking (SMC) [14] replaces numerical modelbased procedures with empirical testing of formalised properties. The original SMC algorithms target fully observable stochastic systems with little nondeterminism and may require the generation of large numbers of sample trajectories from a complete system model. SMC techniques have been utilised to tackle verification of black box probabilistic systems [19], with no model of the system available, but this approach requires large amounts of data. Extensions towards the inclusion of non-determinism have been studied in [12], with preliminary steps towards Markov decision processes. Related to SMC techniques, $[6,15]$ assume the system is encompassed by a finite-state Markov chain and efficiently use data to learn and verify the corresponding model. Similarly, [2, 4] employ machine learning techniques to infer finite-state Markov models from data over given logical formulae.

Bayesian inference uses Bayes theorem to update the probability distribution of a set of hypotheses based on observed data [3]. Bayesian inference for learning transition probabilities in Markov Processes is presented in [16]. 


\section{Background}

\subsection{Parametrised Markov chains - syntax and semantics}

Let $S$ be a finite, non-empty set of states representing all possible configurations of the system being modelled. A discrete-time Markov chain (DTMC) is a stochastic time-homogeneous process over this set of states [1], as follows.

Definition $1 A$ discrete-time Markov chain $\mathbf{M}$ is a tuple $\left(S, \mathbb{T}, \iota_{\text {init }}, \mathrm{AP}, L\right)$, where $S$ is a finite, non-empty set of states, $\mathbb{T}: S \times S \rightarrow[0,1]$ is the transition probability function such that for $\forall s \in S: \sum_{s^{\prime} \in S} \mathbb{T}\left(s, s^{\prime}\right)=1$. The function $\iota_{\text {init }}: S \rightarrow[0,1]$ denotes an initial probability distribution over the states $S$, such that $\sum_{s \in S} \iota_{\text {init }}(s)=1$. The states in $S$ are labelled with atomic propositions $a \in \mathrm{AP}$ via the labelling function $L: S \rightarrow 2^{\mathrm{AP}}$.

Consider the evolution of a Markov chain over a time horizon $t=0,1, \ldots, N_{t}$, with $N_{t} \in \mathbb{N}$. Then an execution of the process is characterised by a state trajectory given as $\left\{s_{t} \mid t=0,1, \ldots, N_{t}\right\}$. The transition function $\mathbb{T}\left(s, s^{\prime}\right)$ specifies for each state $s$ the probability of moving to $s^{\prime}$ in one step, and hinges on the Markov Property, which states that the conditional probability distribution of the future possible states depends only on the current state, namely $\mathbb{P}\left(s^{\prime}=s_{t+1} \mid\right.$ $\left.s_{t}, \ldots s_{0}\right)=\mathbb{P}\left(s^{\prime}=s_{t+1} \mid s_{t}\right)$. Furthermore, the definition of $\mathbf{M}$ requires $\mathbb{T}$ is time homogeneous, that is $\mathbb{P}\left(s^{\prime}=s_{t+1} \mid s_{t}=s\right)=\mathbb{P}\left(s^{\prime}=s_{t} \mid s_{t-1}=s\right), \forall t \in \mathbb{N}$. The model is extended with (internal) non-determinism in order to express lack of complete knowledge of the underlying system.

Definition $2 A$ discrete-time Parametric Markov chain is defined as a tuple $\mathbf{M}_{\Theta}=\left(S, \mathbb{T}_{\theta}, \iota_{\text {init }}, \mathrm{AP}, L, \Theta\right)$ where $S, \iota_{\text {init }}, \mathrm{AP}, L$ are as in Definition 1. The entries in $\mathbb{T}_{\theta}$ are specified in terms of parameters, collected in a parameter vector $\theta \in \Theta$, where $\Theta$ is the set of all possible evaluations of $\theta$. Each evaluation gives rise to an induced Markov chain $\mathbf{M}(\theta)$.

Note we require a certain type of well-posedness of the parameterisation, we demand $\forall s \in S, \forall \theta \in \Theta: \sum_{s^{\prime} \in S} \mathbb{T}_{\theta}\left(s, s^{\prime}\right)=1$. More precisely, any $\theta \in \Theta$, induces a Markov chain $\mathbf{M}(\theta)$ where the transition function $\mathbb{T}_{\theta}$ can be represented by a stochastic matrix. Note also, we assume a distribution on the parameters of the model.

We considered two types of parameterised Markov chain. We use the first, simpler type, as a base case to build the method for the more complex linearly parameterised Markov chains.

1. basic parameterised Markov chains with independently parameterised transition probabilities. Consider $\mathbf{M}_{\Theta}=\left(S, \mathbb{T}_{\theta}, \iota_{i n i t}, \mathrm{AP}, L, \Theta\right)$ with $\Theta \subseteq[0,1]^{n}$ and parameter vector $\theta:=\left(\theta_{1}, \ldots, \theta_{n}\right) \in \Theta$ build up based on individual parameters $\theta_{i} \in[0,1]$. Then the parameterised $\mathrm{MC}$ is considered basic if transition probabilities between states are either known and considered constant with a value in $[0,1]$, or have a single parameter $\theta_{i}\left(\right.$ or $\left.1-\theta_{i}\right)$ associated to them and $\forall s \in S, \forall \theta \in \Theta: \sum_{s^{\prime} \in S} \mathbb{T}_{\theta}\left(s, s^{\prime}\right)=1$ (cf. Fig. 1, left). 

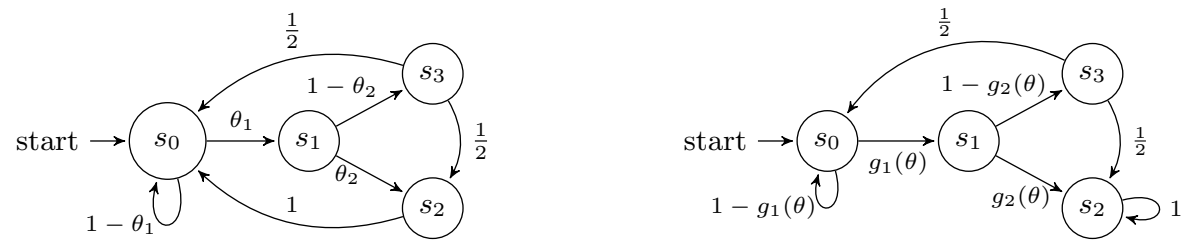

Fig. 1: Two parameterised Markov chains. The nodes of the graph represent states. The labels over the edges provide the probability of taking a transition. The left graph gives parameterised $\mathrm{MC}$ with a basic parameterisation, where the parameters $\theta_{1}, \theta_{2}$ are encompassed in the vector $\theta=\left(\theta_{1}, \theta_{2}\right) \in \Theta=[0,1]^{2}$. The right graph has a linear parameterisation, characterised by affine functions $g_{1,2}: \theta \mapsto[0,1]$.

2. linearly parameterised Markov chains, where unknown transition probabilities can be linearly related. Given $\Theta \subseteq[0,1]^{n}$ and parameter vector $\theta:=$ $\left(\theta_{1}, \ldots, \theta_{n}\right) \in \Theta$ with $\theta_{i} \in[0,1]$, the parameterised MC is considered linearly parameterised if there exists a set of affine functions $g_{l}(\theta):=k_{0}+$ $k_{1} \theta_{1}+\ldots+k_{n} \theta_{n}$ with $k_{i} \in[0,1]$ and $\sum k_{i} \leq 1$, denoted $g_{l}(\theta)_{l \in \mathcal{L}}$. All outgoing transition probabilities of states (or, graphically labels of outgoing edges of a node, cf. Fig.1) have probability $g_{l}(\theta)$ or $1-g_{l}(\theta)$ and $\forall s \in S, \forall \theta \in \Theta: \sum_{s^{\prime} \in S} \mathbb{T}_{\theta}\left(s, s^{\prime}\right)=1$.

The basic case leads to simple procedures, and in Section 5 we develop the linear structure for Bayesian verification. Parameterisations beyond these two categories, such as non-linear ones, are out of the scope of this paper.

\subsection{Properties - Probabilistic Computation Tree Logic}

We consider system requirements specified in probabilistic logics. As we leverage PRISM's parametric model checking tool [10] for synthesis, we can consider the set of properties supported by the synthesis tool: non-nested Probabilistic Computational Tree Logic (PCTL) [1] formulae. For instance, $\mathbb{P}_{\geq 0.5}$ (stay $\mathcal{U}$ get) expresses the property "the probability of remaining in a state labelled with atomic proposition 'stay' until we reach a state labelled as 'get', is bigger or equal to 0.5". PRISM also supports nested PCTL with some restrictions, and a planned extension to this work is to use PROPHESY [8] for parameter synthesis, which supports conditional probabilities and unbounded-time properties. We next define PCTL in nexus to finite discrete-time Markov chains:

Definition 3 Let a discrete-time Markov chain be given. Let $\phi$ be a formula interpreted over states $s \in S$, and $\varphi$ be a formula interpreted on paths of the DTMC. Also, let $\bowtie \in\{<, \leq, \geq,>\}, n \in \mathbb{N}, p \in[0,1], c \in A P$. The syntax of $P C T L$ is given by:

$$
\phi:=\text { True }|c| \phi \wedge \phi|\neg \phi| \mathbb{P}_{\bowtie p}(\varphi), \quad \varphi:=\bigcirc \phi \mid \phi \mathcal{U} \phi .
$$


We define the satisfaction function quantifying satisfaction of these properties over the parameter space as follows. We assume it is a measurable function.

Definition 4 Let $\mathbf{M}(\theta)$ be an induced Markov chain of the parametric Markov chain $\mathbf{M}_{\Theta}$ indexed by parameter $\theta \in \Theta$, and let $\phi$ be a formula in PCTL. The satisfaction function $f_{\phi}: \Theta \rightarrow\{0,1\}$, defined as $f_{\phi}(\theta)=1$ if $\mathbf{M}(\theta) \models \phi$, and 0 otherwise.

\subsection{Bayesian inference}

Our method uses Bayesian inference to learn the probability distribution of parameters in our model class as more evidence or data becomes available. Bayesian inference derives the posterior probability distribution from a prior probability and a likelihood function derived from a statistical model for the observed data. Bayes' law states that, given observed data $D$, the posterior probability of a hypothesis $p(H \mid D)$, is proportional to the likelihood $p(D \mid H)$, multiplied by the prior $p(H)$, as

$$
p(H \mid D)=\frac{p(D \mid H) p(H)}{p(D)} .
$$

$D$ comprises batches of traces of specific length generated by Markov chains instantiated over $\Theta$. The denominator in (1) is an integral over the parameter set $\Theta$, which in general requires numerical approximation. Hence it is of interest to seek a conjugate prior $p(H)$ resulting in a closed-form expression for the posterior $p(H \mid D)$ : in this work we make use of the Dirichlet distribution, which is conjugate to the multinomial [3]. When insufficient initial knowledge is available, we choose a non-informative prior, which has minimal influence on the posterior, such as a uniform prior.

\section{Problem statement and overview of the approach}

Consider a partly unknown dynamical system $\mathbf{S}$, and suppose we can gather a limited amount of sample trajectories from this system as data. Assume the knowledge about the system is encompassed within a parametric model class, describing the behaviour of $\mathbf{S}$ up to the unknown parameterisation of some of its transitions. We plan to investigate the following goal: can we efficiently use the gathered data and the model knowledge of $\mathbf{S}$ to formally verify given PCTL properties over $\mathbf{S}$, quantifying a confidence in our assertions?

The three phases of our work are as follows. In the first phase, Sec. 4, we use parameter synthesis to determine a set of feasible parameters for which the system satisfies the given property. The second phase, Sec. 5, uses Bayesian Inference to infer a distribution over the likely value of the parameters given sample data from the system. In the final phase, Sec. 6 , we combine the outputs of parametric inference and parameter synthesis to quantify the confidence that the system verifies a PCTL property of interest. 
Bayesian probability calculus [3] leads to expressing the confidence in a property as a measure of the uncertainty distribution over the synthesised parameter sets. Uncertainty distributions are handled as probability distributions of random variables. Given a specification $\phi$ and a data set $D$, the confidence $\mathbf{S} \models \phi$ can be quantified via inference as $\mathbb{P}(\mathbf{S}|=\phi| D)=\int_{\Theta} f_{\phi}(\theta) p(\theta \mid D) d \theta$, where $\mathbb{P}(\cdot)$ is a probability measure obtained integrating the distribution $p(\cdot)$ of the uncertainty parameter over $\mathbf{M}_{\Theta}$, expressed as the a-posteriori $p(\theta \mid D)$ given the data set $D$ and the uncertainty distribution $p(\theta)$ over the parameter set $\Theta$.

The computation in the third phase is a key challenge for Markov chains with non trivial parameterisation due to the required complex manipulation of Dirichlet posterior distributions. This motivates the introduction of a Markov chain expansion algorithm in Sec. 5.2, which enables us to analytically obtain samples of complex posterior distributions.

\section{Parameter synthesis}

The first phase of our method uses parameter synthesis and, given a property and a parameterised Markov chain, synthesises the feasible set of parameters corresponding to models satisfying the given PCTL property. This corresponds to the set of parameters for which the binary satisfaction function, $f_{\phi}(\theta)=$ $\mathbb{P}(\mathbf{M}(\theta) \models \phi)$, is equal to 1 . We denote this set $\Theta_{\phi}$, namely

$$
\Theta_{\phi}=\{\theta \in \Theta: \mathbf{M}(\theta) \models \phi\} .
$$

We leverage PRISM's parametric model checking functionality based on [11] to perform this synthesis. [11] expresses quantitative specifications as rational functions that are later manipulated. PRISM's parameteric model checking approach can be applied to unbounded until, steady-state probabilities, reachability reward and steady-state reward properties for parametric DTMCs. The result is a mapping from hyper-rectangles (subsets of parameter valuations) to functions over the parameters.

Alternatives to these techniques have not shown to be scalable or sufficiently general. [5] explores the parameter space with the objective of model verification. [13] employs an analytical approach to parameter synthesis for probabilistic transition systems and is bound to at most two parameters. [7] employs a language-theoretical approach based on regular expressions, which however does not scale as the number of transitions of the Markov model increases. [18] synthesise single-parameter Markov models via accurate interval propagation.

\section{Bayesian inference in parameterised Markov chains}

In this section we consider the application of Bayesian inference to parameterised Markov chains, in order to infer unknown parameter probabilities based on observed data. We will first present the technique for basic parameterised 
Markov chains, and then extend the method to linearly related parameterisations in Sec. 5.2, where we show data obtained from a linearly parameterised Markov chain can equally be represented by data complemented with a set of hidden (or unobserved) data of a basic Markov chain. We use $\mathbb{P}(\cdot)$ to denote a probability measure, and $p(\cdot)$ to denote a probability density function.

\subsection{Basic parameterised Markov chains}

Let us consider a basic parameterised Markov chain $\mathbf{M}_{\Theta}=\left(S, \mathbb{T}_{\theta}, \iota_{i n i t}, \mathrm{AP}, L, \Theta\right)$ (cf. Definition 2). In this basic parameterised Markov chain, every individual parameter $\theta_{i}$ of vector $\theta=\left(\theta_{1}, \theta_{2}, \ldots, \theta_{n}\right) \in \Theta$ is exclusively used to assign the outgoing transition probabilities of a single state. We can decompose our parameter vector $\theta$ into sub-vectors $\theta_{s_{i}}$, giving the parameters for the outgoing transitions of the corresponding state $s_{i}$.

Consider the parameter vector composed of one parameter, $\theta_{s_{k}}=\theta_{j}$, and the corresponding state $s_{k} \in S$, with outgoing transitions $\theta_{j}$ and $1-\theta_{j}$ to states $s_{1}$ and $s_{2}$, respectively. We denote by $p\left(\theta_{j}\right)$ the prior over $\theta_{j}$, which fully defines the transition probabilities $\mathbb{T}_{\theta}\left(s_{k}, \cdot\right)$ at state $s_{k}$. Denote a data set $D$ giving transition counts for trajectories generated from the real system $\mathbf{S}$. For any pair $\left(s_{k}, s_{l}\right) \in S \times S$ the number of transitions $s_{k} \rightarrow s_{l}$ in $D$ is denoted as $D_{s_{k}}^{s_{l}}$. The posterior density $p\left(\theta_{j} \mid D\right)$ over $\theta_{j}$ based on $D$ is

$$
p\left(\theta_{j} \mid D\right)=\frac{\mathbb{P}\left(D \mid \theta_{j}\right) p\left(\theta_{j}\right)}{\mathbb{P}(D)}=\frac{p\left(\theta_{j}\right) \prod_{s^{\prime} \in S} \mathbb{T}_{\theta}\left(s_{k}, s^{\prime}\right)^{D_{s_{k}}^{s^{\prime}}}}{\mathbb{P}\left(D_{s_{k}}\right)}
$$

and depends only on $D_{s_{k}}=\left\{D_{s_{k}}^{s^{\prime}}\right\}_{s^{\prime} \in S}$, i.e., the counts of transitions leaving state $s_{k}$. Note the likelihood function $\prod_{s^{\prime} \in S}\left(\mathbb{T}_{\theta}\left(s_{k}, s^{\prime}\right)\right)^{D_{s_{k}}^{s^{\prime}}}$ takes the form of a multinomial distribution, ${ }^{3}$ which reduces to a binomial in the case of two outgoing transitions. A closed-form expression for the posterior is obtained by taking a conjugate prior, which, for the class of multinomial distributions, is a Dirichlet distribution. For the pair $\left(\theta_{j}, 1-\theta_{j}\right)$ the Dirichlet distribution with hyperparameters $\alpha=\left(\alpha_{1}, \alpha_{2}\right)$ has a probability density function given by

$$
\operatorname{Dir}\left(\theta_{j} \mid \alpha\right)=\frac{1}{B(\alpha)} \theta_{j}^{\alpha_{1}-1}\left(1-\theta_{j}\right)^{\alpha_{2}-1}
$$

on the open simplex defined by $0<\theta_{j}<1$. The normalising constant, $B(\alpha)$, is a multinomial beta function, and can be written in terms of gamma functions as $B(\alpha)=\Gamma\left(\alpha_{1}\right) \Gamma\left(\alpha_{2}\right) / \Gamma\left(\alpha_{1}+\alpha_{2}\right)$. Hence, for a prior $p\left(\theta_{j}\right)=\operatorname{Dir}\left(\theta_{j} \mid \alpha\right)$ we obtain the posterior distribution for $\theta_{j} \sim p\left(\theta_{j} \mid D\right)=\operatorname{Dir}\left(\theta_{j} \mid D_{s_{k}}+\alpha\right)$, namely

$$
p\left(\theta_{j} \mid D\right) \propto p\left(\theta_{j}\right) \prod_{s^{\prime} \in S} \mathbb{T}_{\theta}\left(s_{k}, s^{\prime}\right)^{D_{s_{k}}^{s^{\prime}}} \propto \theta_{j}^{\alpha_{1}-1}\left(1-\theta_{j}\right)^{\alpha_{2}-1} \theta_{j}^{D_{s_{k}}^{s_{1}}}\left(1-\theta_{j}\right)^{D_{s_{k}}^{s_{2}}}
$$

where the normalisation constant of the obtained Dirichlet distribution is $B(\alpha+$ $\left.D_{s_{k}}\right)=\Gamma\left(\alpha_{1}+D_{s_{k}}^{s_{1}}\right) \Gamma\left(\alpha_{2}+D_{s_{k}}^{s_{2}}\right) / \Gamma\left(\alpha_{1}+D_{s_{k}}^{s_{1}}+\alpha_{2}+D_{s_{k}}^{s_{2}}\right)$. In other words, as

${ }^{3}$ A multinomial is defined by its density function $f(\cdot \mid p, N) \propto \prod_{i=1}^{k} p_{i}^{n_{i}}$, for $n_{i} \in$ $\{0,1, \ldots, N\}$ and such that $\sum_{i=1}^{k} n_{i}=N$, where $N \in \mathbb{N}$ is a parameter and $p$ is a discrete distribution over $k$ outcomes. 
data is gathered, we analytically update the posterior probability distribution $p\left(\theta_{j} \mid D\right)$ by updating the parameters of a Dirichlet distribution.

This result can be extended to the case of a state $s_{l}$ with $m>2$ outgoing transitions. We parameterise the outgoing transitions with the sub-vector $\theta_{s_{l}}=$ $\left(\theta_{1}, \ldots, \theta_{m-1}\right)$ and $1-\theta_{1}-\ldots-\theta_{m-1}$, and obtain the posterior for the subvector, $p\left(\theta_{s_{l}} \mid D\right)$. The likelihood function takes the form of an $m$-dimensional multinomial distribution, and we express the prior as an $m$-dimensional Dirichlet.

This yields a posterior distribution as an $m$-dimensional Dirichlet distribution, $p\left(\theta_{s_{l}} \mid D\right)=\operatorname{Dir}\left(\theta_{s_{l}} \mid D_{s_{l}}+\alpha\right)$.

The posterior distribution for the entire parameter vector $p(\theta \mid D)$ is equal to the product of the posterior distributions for the sub-vectors of $\theta$. This holds due to the stated independence of the parameters in a basic parameterised Markov chain, which results in independent priors and independent likelihood functions. Hence $p(\theta \mid D)=\prod_{s_{i}} \operatorname{Dir}\left(\theta_{s_{i}} \mid D_{s_{i}}+\alpha\right)$.

Transition grouping. For simplicity, given a state with multiple outgoing transitions we may obtain the distribution for each parameter using marginal distributions. Consider state $s_{l}$ with $m>2$ outgoing transitions, parameterised with the sub-vector $\theta_{s_{l}}=\left(\theta_{1}, \ldots, \theta_{m-1}\right)$ and $1-\theta_{1}-\ldots-\theta_{m-1}$ We have shown earlier that, if the parameters are independent, the joint posterior distribution over the transition probabilities for this state is an $m$-dimensional Dirichlet: $p\left(\theta_{s_{l}} \mid D\right)=\operatorname{Dir}\left(\theta_{s_{l}} \mid D_{s_{l}}+\alpha\right)$. The marginal distribution of $\theta_{i}$ is a 2-dimensional Dirichlet, or a beta distribution, $\theta_{i} \sim \operatorname{Dir}\left(\alpha_{i},\left(\sum_{i=1}^{m} \alpha_{i}\right)-1\right)$. We can hence obtain a posterior distribution for each parameter, by effectively grouping the training data together for all transitions except the one we obtain the posterior distribution for.

\subsection{Linearly parameterised Markov chains}

In this section we build on the Bayesian inference for basic parameterisations and tackle linearly parameterised Markov chains. As defined before, in a linear parameterised Markov chain, the transition probabilities will be expressed in the form $g(\theta)=k_{0}+k_{1} \theta_{1}+\ldots+k_{n} \theta_{n}$. For a given data set $D$ and a linearly parameterised Markov chain we want to use Bayesian inference to get the posterior distribution $p(\theta \mid D)$ over the parameter set $\Theta$. In order to work with linear parameters we introduce two types of transformations of the Markov chain. In the first, we consider a compression of the data. When two states of the DTMC have "similar" transitions, what can be learned is equivalent. These states are referred to as being parameter similar and will be introduced more precisely in the following. Next we show that, by introducing additional, non-observed states, into the Markov chain and the data, the linear parameterised Markov chain can be transformed to a basic Markov chain with unobserved states (and hidden data). After these transformations we can apply the Bayes rule over the expanded Markov chain and hidden data. 
Parameter similar states. If we have the same parameter appearing multiple times in our Markov chain, we must combine the data obtained from all these transitions to obtain a sole posterior distribution for the parameter in our confidence computation. This technique, referred to as "parameter tying", is used in [17]. We can perform this step analytically for Dirichlet distributions over parameter similar states, by which we denote states with outgoing transitions having identical parameterisations.

Manipulating posterior Dirichlet distributions is mathematically complex because of the dependence between the variables. However, if states are parameter similar, we can use the result in (3). Consider two parameter similar states, $s_{1}$ and $s_{2}$, with outgoing transition probabilities $\theta_{j}$ and $1-\theta_{j}$, and observed data over the transitions. We combine the data to give one posterior Dirichlet distribution for the parameter, $p\left(\theta_{j}\right)=\operatorname{Dir}\left(D_{s_{1}}+D_{s_{2}}+\alpha_{s_{1}}\right)$.

Parameterised Markov chain state expansions. Consider a parameterised DTMC $\mathbf{M}_{\Theta}=\left(S, \mathbb{T}, \iota_{i n i t}, \mathrm{AP}, L, \Theta\right)$. We wish to define a new parameterised DTMC $\mathbf{M}_{\Theta}^{*}$ that produces the same output for our method, but which has a simpler parameterisation. Our method hinges on obtaining a distribution for $\theta$ based on collected training data $D$, and so if $\mathbf{M}_{\Theta}^{*}$ is equivalent to $\mathbf{M}_{\Theta}$, the probabilities of reaching a set of states in $\mathbf{M}_{\Theta}$ must be the same as reaching the equivalent states in $\mathbf{M}_{\Theta}^{*}$, but we may disregard the length of associated paths.

Before introducing the definition of state expansion, we first need to define hidden data. Suppose the two Markov chains have states $S$ and $S^{*}$, such that $S \subset S^{*}$ : all states of $S^{*}$ not in $S$ are defined as hidden. $\Omega$ denotes the set of finite paths $\omega$ in $\mathbf{M}_{\Theta}$, and $\Omega^{*}$ denotes the set of finite paths $\omega^{*}$ in $\mathbf{M}_{\Theta}^{*}$. Then any observed state sequence consists only of states in $S$, and the states in $S^{*} \backslash S$ remain hidden from the observations. The data set $D$ over the states $S$ consists of transition counts $D_{s_{k}}^{s_{l}}$ for pairs $s_{k}, s_{l} \in S$. Observe that for the set of states $S^{*}$ the data is incomplete, namely it does not represent the actual state transitions but only the observed ones. For an observed transition count $D_{s_{k}}^{s_{l}}$, we introduce the extended set $D_{s_{k}}^{s_{l}}$ * as the collection of counts over all hidden paths from $s_{k}$ to $s_{l}$. Consider states $s_{0}$ and $s_{2}$, and hidden state $s_{0}^{*}$ in Figure 3a: hidden paths from $s_{0}$ to $s_{2}$ can be of the form $\left\{s_{0}, s_{2}\right\},\left\{s_{0}, s_{0}^{*}, s_{2}\right\} \in \Omega^{*}$, with the associated extended data count $D_{s_{0}}^{s_{2} *}:=\left\{D_{s_{0}}^{s_{2}}, D_{s_{0}}^{s_{0}^{*}}, D_{s_{0}^{*}}^{s_{2}}\right\}$. The set of possible extended transition counts is denoted as $\mathcal{D}_{s_{k}}^{s_{l}}$ * for the pair $\left(s_{k}, s_{l}\right)$, and $\mathcal{D}^{*}$ for all transitions - note they are set-valued mappings of $D_{s_{k}}^{s_{l}}$ and $D$, respectively.

Definition 5 Consider parameterised Markov chains $\mathbf{M}_{\Theta}=\left(S, \mathbb{T}, \iota_{\text {init }}, \mathrm{AP}, L, \Theta\right)$ and $\mathbf{M}_{\Theta}^{*}=\left(S^{*}, \mathbb{T}^{*}, \iota_{i n i t}^{*}, \mathrm{AP}, L^{*}, \Theta\right)$, both over set $\Theta$. We say $\mathbf{M}_{\Theta}^{*}$ is an expansion of $\mathbf{M}_{\Theta}$ if, for all $D$ and for all $\theta \in \Theta$,

$$
\mathbb{P}_{\mathbf{M}(\theta)}(D)=\mathbb{P}_{\mathbf{M}^{*}(\theta)}\left(\mathcal{D}^{*}\right)
$$

and if $\iota_{\text {init }}=\iota_{\text {init }}^{*}$. The extended labelling map $L^{*}$ is a trivial extension of $L$, assigning labels $L(s)$ for $s \in S$ and an empty label to $S^{*} \backslash S$. 
Theorem 1. The expansion relation is transitive; if $\mathbf{M}_{\Theta, 1}, \mathbf{M}_{\Theta, 2}, \mathbf{M}_{\Theta, 3}$ are all parameterised with $\Theta, \mathbf{M}_{\Theta, 3}$ is an expansion of $\mathbf{M}_{\Theta, 2}$ and $\mathbf{M}_{\Theta, 2}$ is an expansion of $\mathbf{M}_{\Theta, 1}$, then $\mathbf{M}_{\Theta, 3}$ is an expansion of $\mathbf{M}_{\Theta, 1}$.

Case I: Transition splitting. We split a transition probability parameterised with $k_{0}+\sum_{i} k_{i} \theta_{i}$ into transitions to hidden states with probabilities $k_{i} \theta_{i}$, and refer to this operation as transition splitting. As a basic example, consider Fig. 2 where state $s_{0}$ in $M$ has two outgoing transition probabilities expressed as functions of the parameter vector, $g(\theta)$ and $1-g(\theta)$, where $g(\theta)=k_{0}+k_{1} \alpha+k_{2} \beta$. We expand $\mathbf{M}_{\Theta}$ into $\mathbf{M}_{\Theta}^{*}$ by splitting state $s_{1}$ into a set of states, and splitting the transition from $s_{0} \rightarrow s_{1}$ into the monomials concerning each parameter in $\theta$, as shown in Fig. 2. $\mathbf{M}_{\Theta}^{*}$ is an expansion of $\mathbf{M}_{\Theta}$ as per Def. 5 .

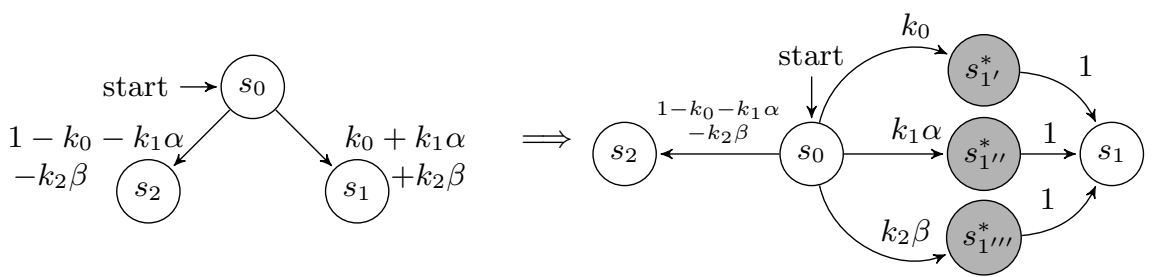

Fig. 2: Case I: Transitions splitting

Lemma 1. Transition splitting of $\mathbf{M}_{\Theta}$ (Case I) generates an expansion of $\mathbf{M}_{\Theta}$.

Case II: State splitting. We present a second case, state splitting, for a parameter $\theta_{i}$ multiplied by a constant, $k_{i} \theta_{i}$. Consider the simple DTMC in Fig.3a, and the state $s_{0}$ in $\mathbf{M}_{\Theta}$ with two outgoing transition probabilities expressed as a constant multiplied by one parameter, $k_{1} \theta_{1}$ and $1-k_{1} \theta_{1}$, where $0 \leq k_{1} \leq 1$. We expand $\mathbf{M}_{\Theta}$ to give $\mathbf{M}_{\Theta}^{*}$ by splitting state $s_{0}$ into two states, and compute the transition probabilities the imposed expansion demands. As an additional ex-

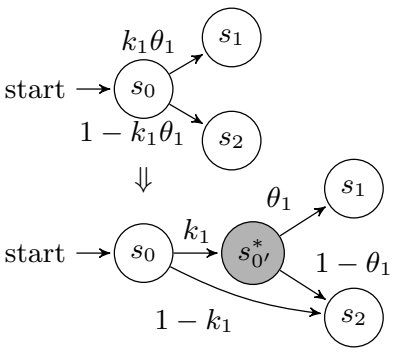

(a) Simple example of state splitting

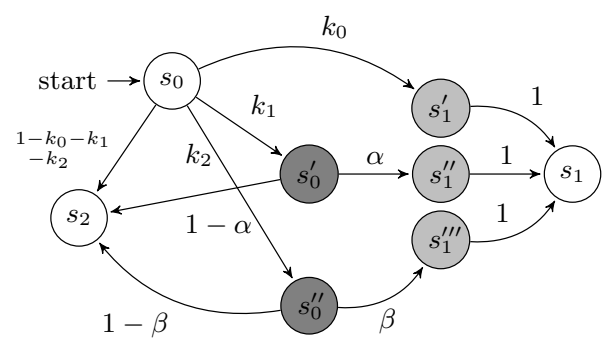

(b) State splitting of Fig.2 (cf. Case I).

Fig. 3: Case II: state splitting (two examples)

ample, notice the transitions studied in Case I are all of the form $k_{i} \theta_{i}$. Applying the state splitting to this expanded DTMC we obtain Fig. 3b. The subsequent 
application of both state splitting cases (cf. Fig. 3b) induces again an expanded parameterised Markov chain as per Def. 5 .

Lemma 2. State splitting of $\mathbf{M}_{\Theta}$ (Case II) generates an expansion of $\mathbf{M}_{\Theta}$.

We are led to the following result.

Theorem 2. Any linearly parameterised Markov chain can be expanded into a basic parameterised Markov chain by application of Lemma 1 and 2.

Bayesian inference with missing data We now consider Bayesian inference on the newly expanded Markov chain $\mathbf{M}_{\Theta}^{*}$. The data set $D$, which is sampled from our system, corresponds to a state trajectory or set of trajectories over the model $\mathbf{M}_{\Theta}$. This set further comprises only part of the corresponding trajectories in the expanded model $\mathbf{M}_{\Theta}^{*}$. For a given trajectory in $D$, we refer to $D^{*}$ as the completed trajectory, and to $\mathcal{D}^{*}$ as the set of all possible completions $D^{*}$. Note the expanded parametric Markov chain has a basic parameterisation, hence for a given completed data set $D^{*}$ the Bayes rule as elaborated in (1) can be applied to obtain $p\left(\theta \mid D^{*}\right)$. For $\mathbf{M}_{\Theta}^{*}$ Bayes rule can be applied over the hidden data as follows:

$$
\begin{aligned}
p(\theta \mid D) & =\frac{\sum_{D^{*} \in \mathcal{D}^{*}} p\left(\theta, D^{*}, D\right)}{\mathbb{P}(D)}=\frac{\sum_{D^{*} \in \mathcal{D}^{*}} p\left(\theta \mid D^{*}, D\right) \mathbb{P}\left(D^{*} \mid D\right) \mathbb{P}(D)}{\mathbb{P}(D)} \\
& =\sum_{D^{*} \in \mathcal{D}^{*}} p\left(\theta \mid D^{*}\right) \mathbb{P}\left(D^{*} \mid D\right) .
\end{aligned}
$$

Completed data sets have a multinomial distribution dependent on the parameterisation, hence the distribution of $D^{*}$ is given as $\mathbb{P}\left(D^{*}\right)=\int_{\Theta} \mathbb{P}\left(D^{*} \mid \theta\right) p(\theta) d \theta$. For a given $D$ the conditional distribution $\mathbb{P}\left(D^{*} \mid D\right)$ is $\mathbb{P}\left(D^{*} \mid D\right)=\mathbb{P}\left(D^{*}\right) / \mathbb{P}(D)$, with $D^{*} \in \mathcal{D}^{*}$ and $\mathbb{P}(D)=\sum_{\mathcal{D}^{*}} \int_{\Theta} \mathbb{P}\left(D^{*} \mid \theta\right) p(\theta) d \theta$.

Remark 1. Realisations of the posterior can be obtained without computing the entire integral as follows. A set of realisations $\theta_{i}$ for $i \in\{1, \ldots, \mathcal{N}\}$ with probability density function $p(\theta \mid D)$ can be obtained by generating samples $D_{i}^{*}$ with distribution $\mathbb{P}\left(D^{*} \mid D\right)$ and subsequently generating samples $\theta_{i}$ with distribution $p\left(\theta \mid D_{i}^{*}\right)$ for all $i \in\{1, \ldots, \mathcal{N}\}$. These samples can then directly be used to perform the confidence calculation as in Sec. 6 .

Algorithm 1 presents the state expansion procedure, and Algorithm 2 in the next section summarises how to obtain a realisation of the posterior $p\left(\theta \mid D^{*}\right)$, and to integrate it with the confidence computation.

\section{Bayesian verification: computation of confidence}

In this section we detail the final phase of our method: a quick procedure computes a confidence estimate for the satisfaction of a PCTL specification formula $\phi$ by a system $\mathbf{S}$ of interest, namely $\mathbf{S}=\phi$. Our method takes as input a posterior distribution over $\Theta$, obtained using Bayesian inference in Sec. 5.2, and the feasible set for the parameters, obtained by parameter synthesis in Sec. 4. 


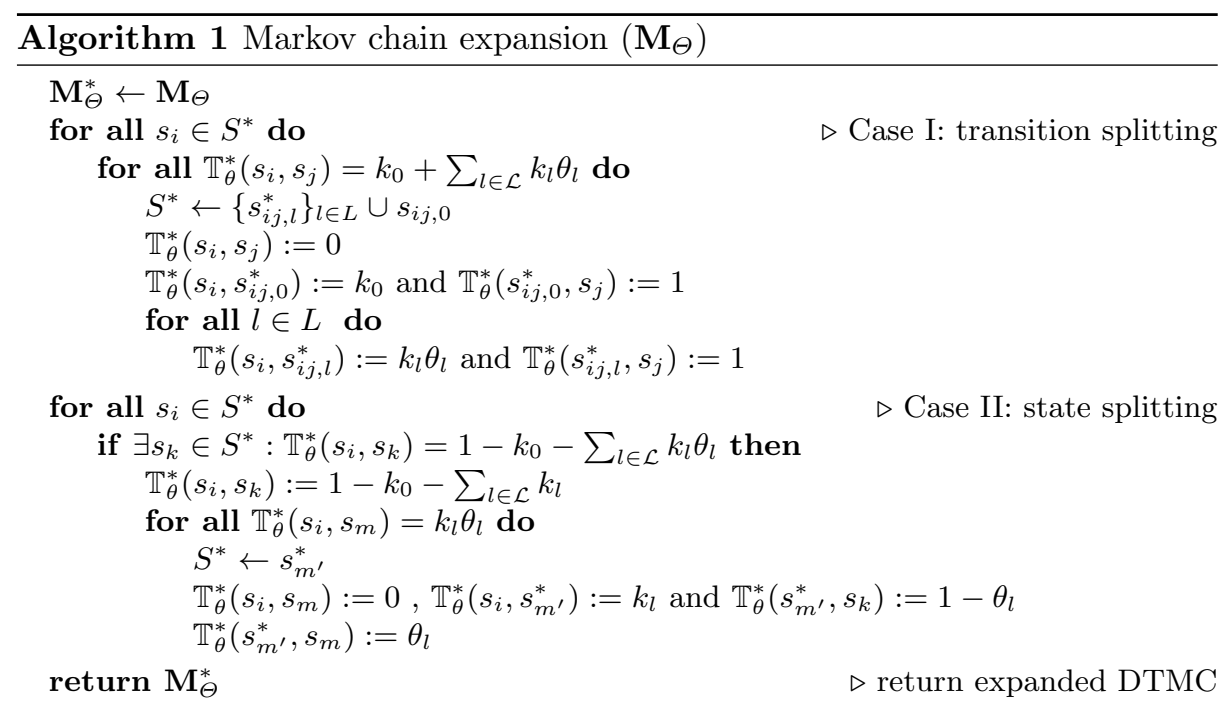

Definition 6. Given a PCTL specification $\phi$, a complete trace (sample trajectory) $D$ of the system $\boldsymbol{S}$ up to time $t$, and a transition function $\mathbb{T}$, the confidence $\boldsymbol{S} \models \phi$ can be quantified by Bayesian Inference as

$$
\mathbb{P}(\boldsymbol{S} \models \phi \mid D)=\int_{\Theta} f_{\phi}(\theta) p(\theta \mid D) d \theta .
$$

As we only consider the satisfaction of a property $\mathbf{S} \models \phi$ as a binary-valued mapping from the space of parameters, the satisfaction function in $(4), f_{\phi}$ : $\Theta \rightarrow\{0,1\}$, (4) can be reformulated as:

$$
\mathbb{P}(\mathbf{S}|=\phi| D)=\int_{\Theta_{\phi}} p(\theta \mid D) d \theta,
$$

where $\Theta_{\phi}$ denotes the set of parameters corresponding to models verifying the property $\phi$ (as generated by PRISM). Further, given the independent posterior distributions for each parameter in $\theta$ resulting from Sec. 5.2, the confidence can be computed as $\mathbb{P}(\mathbf{S} \models \phi \mid D)=\int_{\Theta_{\phi}} \prod_{\theta_{i} \in \theta} p\left(\theta_{i} \mid D\right) d \Theta$. The integral of a Dirichlet distribution can be obtained by iterative or numerical methods: here we use a simple Monte-Carlo approach, which depends on samples of the posterior distribution as clarified in Algorithm 2.

\section{$7 \quad$ Experiment results}

We show our approach requires smaller amounts of data than statistical model checking (SMC) to verify the system satisfies a given quantitative specification up to a prescribed confidence level. We further claim our approach is more robust than standard SMC in situations where only data of limited trace length is available. 


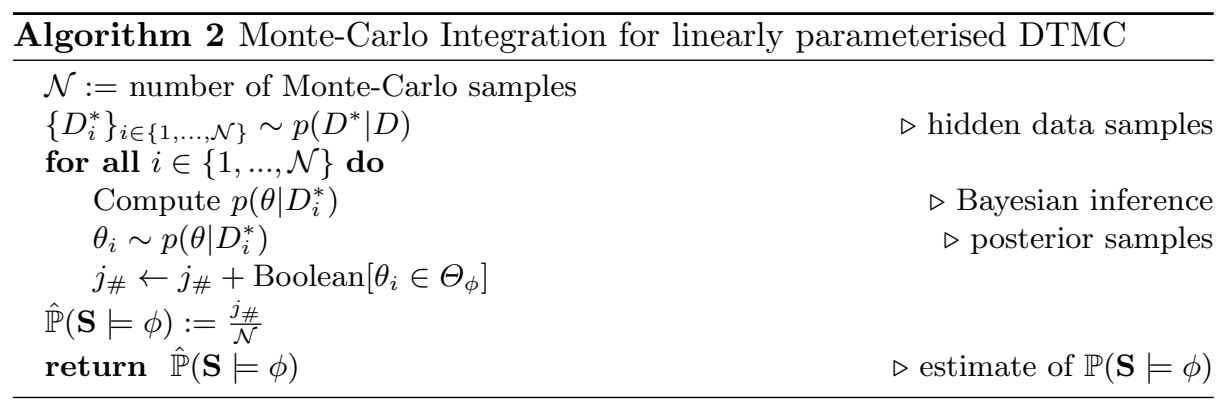

Experiment setup We focus our experimental discussion on the basic parameterised Markov chain $\mathbf{M}_{\Theta}$ in Figure 1 and the PCTL property $\phi=\mathbb{P}_{>0.5}\left[\neg s_{3} \mathcal{U} s_{2}\right]$.

The ground truth for $\mathbf{S}=\mathbf{M}(\theta)$, namely $Y_{\text {true }}$, is a step function over the parameter $\theta$, namely

$$
Y_{\text {true }}= \begin{cases}0 & \text { if } \theta \leq 0.5, \\ 1 & \text { if } \theta>0.5,\end{cases}
$$

so the feasible set is $\Theta_{\phi}=[0.5,1]$. We choose a uniform prior for both methods: for our approach $p(\theta \mid D)=\operatorname{Dir}(1,1)$, which, for property $\phi$, means $p(\mathbf{M}(\theta) \mid=$ $\phi)=\operatorname{Dir}(1,1)$; for $\mathrm{SMC}$ we set $p(\mathbf{M}(\theta) \models \phi)=\operatorname{Dir}(1,1)$. We run both methods over empirical data obtained from $\mathbf{M}(\theta)$, our "underlying system", for values of $0<\theta<1$, i.e., different "underlying systems", and compare the outcomes with the ground truth. We collect data, denoted $D$, from our underlying system in the form of a set of state trajectories of a set length. We vary trajectory length to test robustness to data with incomplete coverage. We disregard the numerical error in the Monte Carlo approximate integration, which is the same for both techniques.

We compute the mean squared error (MSE) between the confidence outcome and the ground truth from Equation (6), namely $M S E=\frac{1}{n} \sum_{i=1}^{n}\left(Y_{\text {true }}-Y_{i}\right)^{2}$, where $n$ is the number of experiments run and $Y_{i}$ is the result $\mathbb{P}\left(\mathbf{M}_{\theta} \models \phi\right)$ for the $i$-th run.

The SMC we compare our work to is "black box" and collects sample trajectories from the system, then determines whether the trajectories satisfy a given property, and applies statistical techniques (such as hypothesis testing) to decide whether the system satisfies the property or not, with some degree of confidence. Our "grey-box" approach collects data from the system, uses the data to determine a distribution over parameter values in the parameterised model class and applies statistical techniques (in this case, a Bayesian confidence calculation) to decide whether the system satisfies the property or not, with some degree of confidence. We could then additionally apply hypothesis testing to our approach. However, as we do not do this, for a meaningful comparison with our approach we implement the framework of the SMC procedure outlined in [14] and omit the hypothesis testing. Instead, we compute a Bayesian confidence by integrating the posterior distribution given over the $[0,1]$ interval, representing the probability of a trace satisfying the property. The trace generation and trace verification 
stages of SMC are implemented in the same way in the four statistical model checking methods in PRISM.

Results and Discussion The first point to note is the confidence is low, and MSE high for parameter values close to $\theta=0.5$ for both approaches. This is due to $\theta=0.5$ being on the edge of the feasible set and is consistent with the information we wish to obtain from the confidence calculation: if the parameter value is near the edge of the feasible set, we need to know its value precisely to be sure it falls in the feasible set. Consider that in order to compute the confidence $\mathbf{S}=\phi$, we integrate the posterior distribution over the feasible set $\Theta_{\phi}=\{\theta>0.5\}$. The posterior distribution for $\theta=0.5$ should have a peak centred at 0.5 with half of the area under the peak in the feasible set, leading to $\mathbb{P}(\mathbf{M}(\theta)=\phi)=0.5$. The height and width of the distribution $p(\theta \mid D)$ are characterised by the amount of data available, as well as the consistency of the data, and so we expect the MSE to be higher for parameter values close to the threshold.

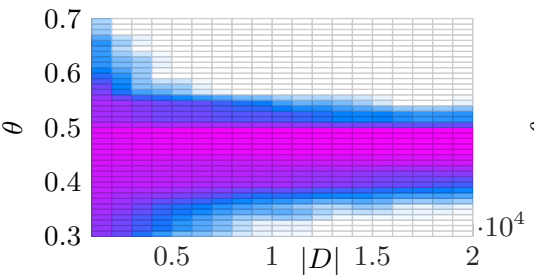

(a) SMC: Trace length 10

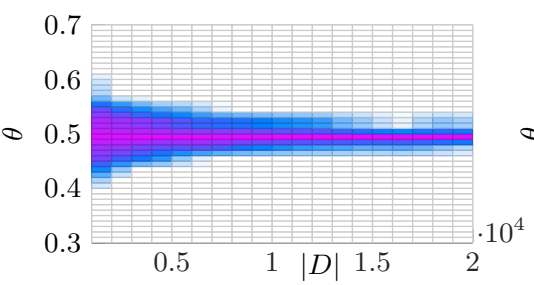

(c) Trace length 10

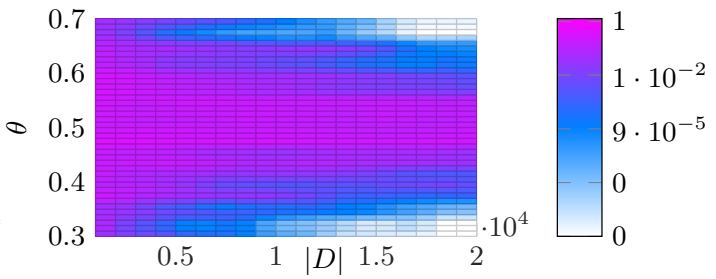

(b) SMC: Trace length 100

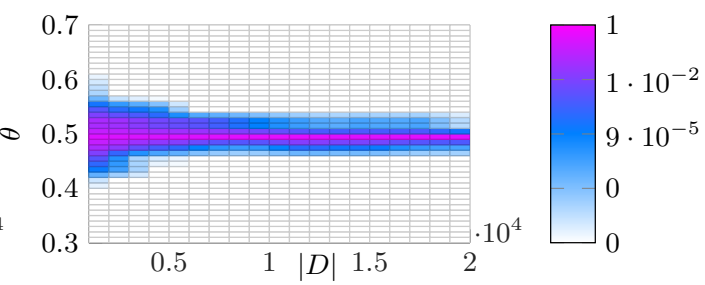

(d) Trace length 100

Fig. 4: Outcomes of SMC are given in (a) and (b), outcomes of our approach are given in (c) and (d). The comparison is done over a data set $D$ composed of traces of 10 and 100 transitions. On the x-axis, $1000 \leq|D t| \leq 20000$. On the y-axis, $0.3 \leq \theta \leq 0.7$. The darker (purple) colour indicates a higher mean squared error.

The key result is, for both approaches, the mean squared error reduces as $|D|$ increases and the variance decreases, but our approach consistently produces a smaller error and variance than SMC for any parameter values excluding $\theta=0.5$ (where both approaches perform comparably). Our approach requires an order of magnitude less data than SMC and above $|D|=2000$, the error for our approach is smaller than the error in the Monte Carlo integration, whereas SMC does not reach this precision threshold in our experiments, which we perform up to $|D|=200000$. 
We ascribe both the reduced error and reduced variance to the data efficiency of our approach: SMC receives the training data in the form of short traces, and discerns whether a trace is a counter example or witness for the property. A trace can, however, be neither, in which case it is discarded, even if that trace contains parameterised transitions. Our approach counts each parameterised transition in the data, and so uses more of the data available than SMC. It is unsurprising accuracy and variance improve when more data is used.

We investigate robustness in a situation where it is only possible to collect short trajectories from the system, whilst verifying an unbounded property. Figure $4 \mathrm{a}$ and Figure $4 \mathrm{~b}$ show the performance of SMC with $|D|$ made up of trace lengths of 10 and 100 transitions respectively. We show a part of our data set, discarding data above $|D|=20,000$ where our approach produces no measurable error. The mean squared error in Figure $4 \mathrm{~b}$ is $50 \%$ lower than in Figure $4 \mathrm{a}$ over the entire parameter range, but the run with trace lengths of 10 performs better for values of $\theta>0.55$.

We explain this because, computed using PRISM, the expected length of a witness for our property and Markov Chain ranges between 4.33, for $\theta=0.3$ and 2.42 for $\theta=0.7$ (due to the symmetrical structure of our Markov Chain, the lengths of counter-examples are also expected to be the same). Thus a large proportion of the traces of length 10 are discarded, and so SMC has less data to use, explaining the increased error across the parameter range. However, when $\theta>0.55$, the expected counter-example length is higher, and so the number of traces of length 10 that are useful begins to exceed the total number of traces of length 100 received.

In contrast, the performance of our approach, shown in Figure 4c and Figure 4d, yields approximately the same outcomes for both trace lengths, as we consider each transition in the training data individually and only discard nonparameterised transitions. Admittedly it is not always the case that the performance of our method is independent of the length of the traces: consider for example the case of a large Markov chain where a parameterised transition is only reachable after a large number of steps. In this case the performance of our approach would be comparable to SMC.

We run experiments on linearly parameterised Markov chains of a similar scale and obtain comparable results.

\section{Conclusions and future work}

We have presented a data-based verification approach addressing incomplete model knowledge. The method offers a framework to integrate Bayesian inference and formal verification, and in comparison to standard statistical model checking promises to be more parsimonious with the required data.

We plan to investigate extensions in the following directions: performing parameter synthesis with alternative available techniques, such as [8], which builds on the work of [10] using graph topological properties and fixed points); working with non-linearly parameterised Markov chains; inspired by [9], integrating 
external non-determinism in the form of actions, thus leading to parameterised Markov decision processes. Finally, we are interested in the use of Bayesian hypothesis testing, which will further solidify this method as a provable verification technique even when the prior probability distribution is not reliably known.

\section{References}

1. Baier, C., Katoen, J.: Principles of model checking. MIT Press (2008)

2. Bartocci, E., Bortolussi, L., Sanguinetti, G.: Learning temporal logical properties discriminating ECG models of cardiac arrhytmias. CoRR abs/1312.7523 (2013)

3. Bernardo, J., Smith, A.: Bayesian Theory. Chichester: Wiley (1994)

4. Bortolussi, L., Sanguinetti, G.: Learning and designing stochastic processes from logical constraints. Logical Methods in Computer Science 11(2) (2015)

5. Brim, L., Ceska, M., Drazan, S., Safránek, D.: Exploring parameter space of stochastic biochemical systems using quantitative model checking. In: CAV. LNCS, vol. 8044, pp. 107-123. Springer (2013)

6. Chen, Y., Nielsen, T.D.: Active learning of Markov decision processes for system verification. In: ICMLA. pp. 289-294. IEEE (2012)

7. Daws, C.: Symbolic and parametric model checking of discrete-time Markov chains. In: ICTAC. LNCS, vol. 3407, pp. 280-294. Springer (2004)

8. Dehnert, C., Junges, S., Jansen, N., Corzilius, F., Volk, M., Bruintjes, H., Katoen, J., Ábrahám, E.: Prophesy: A PRObabilistic ParamEter SYnthesis Tool. In: CAV. LNCS, vol. 9206, pp. 214-231. Springer (2015)

9. Haesaert, S., Van den Hof, P.M.J., Abate, A.: Data-driven property verification of grey-box systems by Bayesian experiment design. In: ACC. pp. 1800-1805. IEEE (2015)

10. Hahn, E.M., Hermanns, H., Wachter, B., Zhang, L.: PARAM: A model checker for parametric Markov models. In: CAV. LNCS, vol. 6174, pp. 660-664. Springer (2010)

11. Hahn, E.M., Hermanns, H., Zhang, L.: Probabilistic reachability for parametric Markov models. In: SPIN. LNCS, vol. 5578, pp. 88-106. Springer (2009)

12. Henriques, D., Martins, J., Zuliani, P., Platzer, A., Clarke, E.M.: Statistical model checking for Markov decision processes. In: QEST. pp. 84-93. IEEE (2012)

13. Lanotte, R., Maggiolo-Schettini, A., Troina, A.: Parametric probabilistic transition systems for system design and analysis. Formal Asp. Comput. 19(1), 93-109 (2007)

14. Legay, A., Delahaye, B., Bensalem, S.: Statistical model checking: An overview. In: RV. LNCS, vol. 6418, pp. 122-135. Springer (2010)

15. Mao, H., Jaeger, M.: Learning and model-checking networks of I/O automata. In: ACML. JMLR, vol. 25, pp. 285-300. JMLR.org (2012)

16. Peter Eichelsbacher, A.G.: Bayesian inference for Markov chains. Journal of Applied Probability 39(1), 91-99 (2002)

17. Poupart, P., Vlassis, N.A., Hoey, J., Regan, K.: An analytic solution to discrete Bayesian reinforcement learning. In: ICML. ACM, vol. 148, pp. 697-704. ACM (2006)

18. Su, G., Rosenblum, D.S.: Nested reachability approximation for discrete-time Markov chains with univariate parameters. In: ATVA. LNCS, vol. 8837, pp. 364379. Springer (2014)

19. Younes, H.L.S.: Probabilistic verification for "black-box" systems. In: CAV. LNCS, vol. 3576, pp. 253-265. Springer (2005) 